\title{
The Effect of Some Sugars on the Growth of Aspergillus niger
}

\author{
Hewa O. HAMAD ${ }^{1 *}$, M. Hakki ALMA ${ }^{2}$, Hero M. ISMAEL ${ }^{3}$, Ali GOCERI ${ }^{2}$
}

\author{
${ }^{1}$ Ministry of Health, KRG, Iraq \\ ${ }^{2}$ KSU, Faculty of Forestry, Dept. Forestry Engineering, Kahramanmaras, Turkey \\ ${ }^{3}$ Salahaddin University, College of Science, KRG, Iraq
}

\author{
Received (Geliş Tarihi): 23.10.2014
}

Accepted (Kabul Tarihi): 23.12.2014

\begin{abstract}
The majority of black Aspergilli, including Aspergillus niger. Here, we provide to evidence that the exploration of sugar uptake in the filamentous fungus A. niger as a sole carbon source. The goal of this research line is determination of the growth of fungi was evaluated every $24 \mathrm{~h}$, measuring the colony diameter $(\mathrm{cm})$. A. niger was inoculated onto two culture media: PDA: for maintains a strain as pure while Czapeck Dox Agar was used in investigation into their carbon requirement, using five different carbon sources (vizs. glucose, fructose, sucrose, maltose, and starch). The fungus was tested grew sparsely on the basal medium lacking in carbon, which was the control. However this fungus was found to vary from their ability to use the supplied sources of carbon. Fructose and sucrose were found to be suitable sources of carbon for a fungal isolates, whereas glucose and maltose proved good carbon source to have a higher affinity. Starch as a polysaccharide, was a poor source of carbon for the growth of this isolate. Despite earlier claims, saccharides rather than monosaccharide were breakdowns extracellularly by means of a broad range of extracellular enzyme activities from Aspergillus niger.
\end{abstract}

Key words: Aspergillus niger, Saccharides utilization, fungal growth

\section{INTRODUCTION}

Fungi are significant environmental microorganisms especially in the ecosystem of the nature where they are responsible for spoilage, production of mycotoxins and in some cases desirable bioconversions. Consequently, it is important to know their requirements for nutrients and carbon-skeleton nutrient, water, temperature, oxygen and other factors for nutrient for fungi and use to energy production, carbohydrates are presented in fungi primarily as saccharide (Hashimoto et al., 2005). Knowledge of structures and properties of sugar are classified as follows: (i) Monosaccharaide: it is available forms of absorption; it cannot be hydrolyzed into simple once, (ii) Disaccharide: it is hydrolyzed into two molecules of monosaccharaides forms, (iii) Oligosaccharide: the number of carbon atoms in their structure is $2-10$ molecules, and (iv) Polysaccharide: it yields more than 10 molecules of monosaccharaides which may be linear or branched (Mcnaught, 1996).

Filamentous fungi represent a physiologically diverse group of micro-organisms. Their growth-form can be long, thin, branched threads of mycelium, but also compact mycelial pellets (Schrickx et al., 1993).

In nature, filamentous fungi are able to utilize a great variety of carbon sources of secreting a wide range of different enzymes in large amounts of their environment (Fang et al., 1998).

Aspergillus niger $\{$ it is a filamentous fungus growing aerobically composed of black-colonies (Frisvad et al., 2002) \} is a mold known for its ability to produce different kinds of enzymes and thus to degrade a big diversity of organic compounds. This characteristic allows the growth of the species in different media and environments (Schuster et al., 2002).
On the other hand, Aspergillus niger is equipped for utilizing sugar as the sole source of carbon and energy for cell growth and metabolism. Growth and allocation of A. niger response to saccharides is well established and generally include increasing entire colonies, biomass, and reducing level of carbohydrates in the surrounding environment (Gupta and Neha, 2012).

In the present study, we describe the influence of various refined carbohydrate sources such as glucose, fructose, maltose, sucrose, and starch on growth and activity by $A$. niger was studied under colony diameter measurement technique.

\section{MATERIALS and METHODS}

Fungus Sample: In this study used A. niger and suitable to select; because it was a widely distributed over nature, easily growth on anywhere area, and produce considerable amount of circular colony. Also, the techniques of isolation, cultivation, and identification were simpler. This fungus was pre-served in Biology Department, College of Science, Salahaddin University, Erbil, Iraq.

Culture Media: Fungus selected depend upon the medium or substrate for synthesis their cellular constituents and obtain necessary energy for their life; the media which was used for the present work purposes includes: Potato Dextrose Agar (this medium was prepared according to the formula developed by Bilgrami and Verma, 1988), PDA is natural medium; and Czapek Dox Agar (this medium was prepared according to the formula developed by Thom and Church, 1926), CDA is synthetic medium and it had a defined chemical composition so that it was recommended for isolation of some fungi with similar physiological requirements (Eaton et al., 1998). The 
medium was sterilized by autoclaving at $121^{\circ} \mathrm{C}$ for 15 minutes. Fungi were plated on the agar medium and incubated at $30^{\circ} \mathrm{C}$ for 5 days and $\mathrm{pH} 5.5$ (Khokhar et al., 2011). Although, fungal isolates could be kept and transferred to another agar plate in order to replenish their nutrient source (Henderson, 1961).

Growth Experiments: Puncture Test Method (it was used to determine the puncture, rupture, and propagation characteristics of a fungus) was used for inoculation. The organisms were growing a circle on modified Czapek Dox Agar after obtained fungus from pure culture of PDA and transferred onto central of a plate. Modified Czapek Dox Agar (NaNO3: 3g, MgS04.7H20: 0.5g, KCl: 0.5g, KH2PO4: $1.0 \mathrm{~g}$, sugar: $30 \mathrm{~g}$, agar: $20 \mathrm{~g}$, water: $1000 \mathrm{ml}$ ) was using different types of sugar (glucose, fructose, maltose, sucrose, and starch) and apply at different concentrations $(7.5 \mathrm{~g} / 250 \mathrm{~mL}$, $3.75 \mathrm{~g} / 250 \mathrm{~mL}$ ). The control was used with no sugar content of a medium (Henderson, 1961).

Estimations of growth: Growth was recorded by measuring the diameter of colonies in two directions at right angles with a rule. Growth rates were calculated for measuring when the colonies were growing at a constant rate (Trinci, 1969).

\section{RESULTS}

On observation in this work, it was apparent that all sugar's type used a deterring effect on the growth of fungal colonies but some saccharides were more effective than another one. There was done statistical analysis among utilization of all sugar substrate.

Typical results of the relation between the rate of $A$. niger growth and monosaccharaides uptake are shown in (Figure 1). Generally, the normal concentration $(7.5 \mathrm{~g}$ sugar/ $250 \mathrm{~mL}$ solution) of fructose was the most useful to enhance fungal growth than glucose. Besides that, utilization of these sugars at half value (3.75 g sugar/ $250 \mathrm{~mL}$ solution) was same result but it was smaller in growth of colony and diameter measurement.

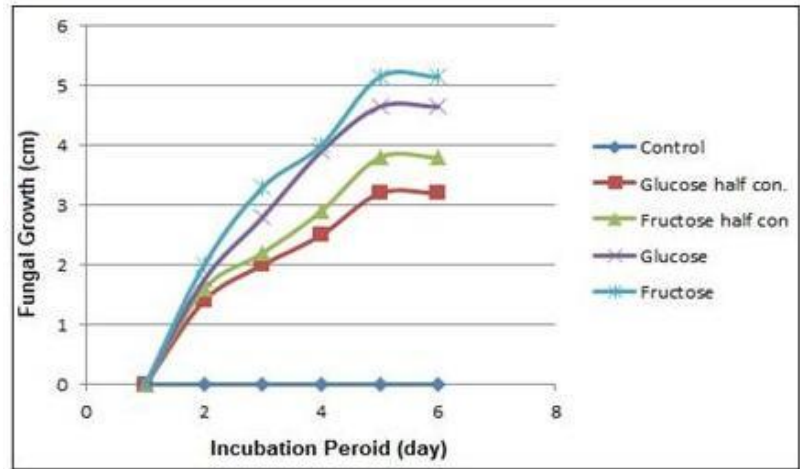

Figure 1. Illustrates the effect of the monosaccharaides on the growth of Aspergillus niger

After incubation $A$. niger with media was containing disaccharides; as shown in (Figure 2). Sugar was splitting may be via hydrolytic processes or enzymatic activity. Sucrose supports maximum growth and good sources of carbon for the growth when compare with maltose utilization at both concentration $(7.5 \mathrm{~g}$ sugar/ $250 \mathrm{~mL}$ solution) and (3.75 g sugar/ $250 \mathrm{~mL}$ solution) but variation occurred in colony diameter.

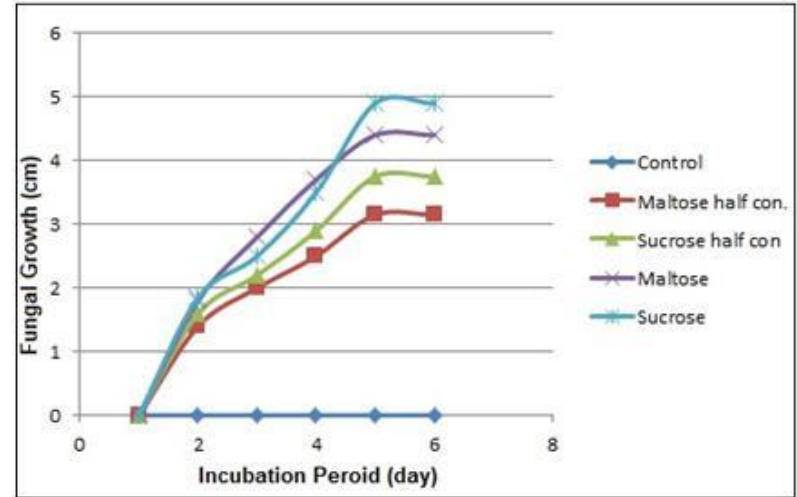

Figure 2. Illustrates the effect of the disaccharides on the growth of Aspergillus niger

In (Figure 3) represent the screening of $A$. niger with the media contained starch as a sloe of carbon sources. Whatsoever, $A$. niger produced $\alpha$-amylase and glucoamylase. The results of both concentrations $(7.5 \mathrm{~g}$ sugar/ $250 \mathrm{~mL}$ solution) and $(3.75 \mathrm{~g}$ sugar/ $250 \mathrm{~mL}$ solution) showed higher enzyme activities but not similar in growth rates and/or mycelium size development.

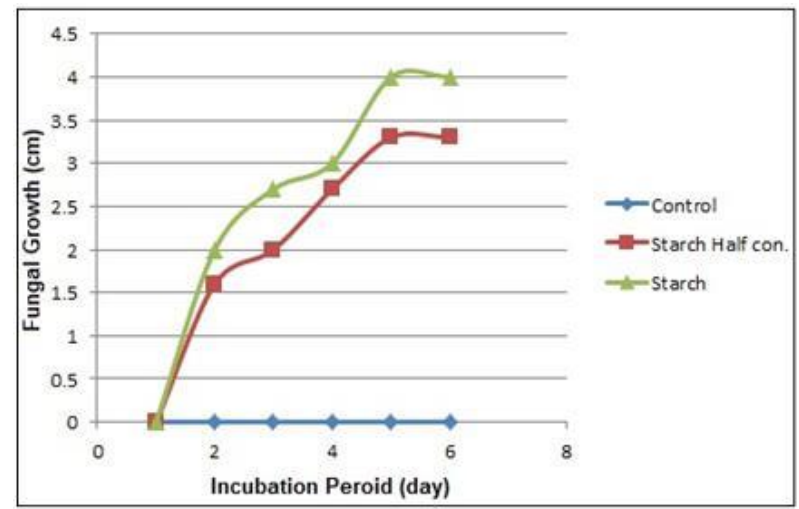

Figure 3. Illustrates the effect of the polysaccharide on the growth of Aspergillus niger

\section{DISCUSSION}

Aspergillus niger is a filamentous fungi. It is able to digest a wide range of carbohydrates when substrate was containing saccharides which available as the sole source of carbon and energy. All the cellular components must be synthesized from these compounds via appropriate metabolic pathways. Among the practical problem that utilization effect of saccharides for energy and as carbon source by fungus, are: (1) physical availability of sugar, (2) cultural condition, and (3) adaptation of the strain on the substrate. 
There are several known transporters capable of transporting the simple sugar such as hexose and pentose into the cell for subsequent phosphorylation and conversion into mainly biomass and $\mathrm{CO} 2$. Moreover, in the study Mchunu et al., (2013) indicates that monosaccharaides showed good assimilation by Trichoderma reesei and Aspergillus niger.

The absorption of the hexoses (glucose and fructose) by A. niger was an active process of the sense that it was dependent on the rate of normal metabolism of the mycelium. Glucose was more rapidly absorbed at all concentrations than fructose. Therefore, the fungus appears to have a higher affinity with glucose than fructose; but the growth rate was often rapid and extensive of fructose as more as glucose. Fructose application had a greatly colony diameter when compared the usage of glucose and the ratio of fructose to glucose several times as much fructose as glucose present; because of during glycolysis, glucose was phosphorylated to form glucose-6-phosphate then by isomerization reaction in which glucose-6-phosphate was converted to fructose-6-phosphate; there was during a time periods here used. In addition, the main destination of fructose absorption was into higher saccharide molecules. There was escaping a part of the conversion: glucose to fructose, so fructose directly made phosphorylation and formed fructose-6-phosphate (Figure 4). In each case, the estimation of colony size of fructose was greater than the glucose in the media was inoculated by $A$. niger.

Mehrotra and Kumar (1961) observed that the carbon utilization studies was a quick approach to studying and assessing filamentous fungi for specific activities and showed that the fructose was earlier and faster according to different behavior from the rest of the organisms; therefore, inducing the highest growth and increasing the biomass of fungi.

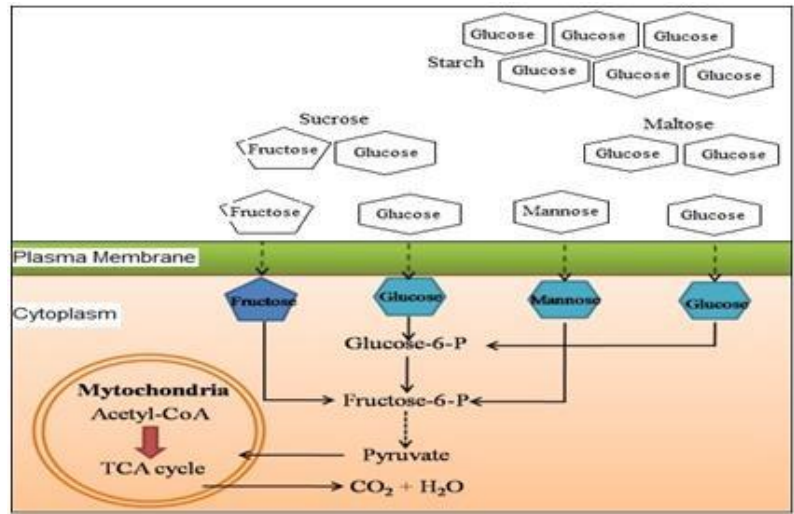

Figure 4. Illustrates the assimilation of simple sugar and cellular respiration

Absorption of disaccharides by A. niger was generally depended upon the production of necessary hydrolytic enzymes. On the other hand, the integration and coordination of metabolic processes in the filamentous fungi, and their modulation in response to environmental and developmental changes, is brought about by regulation of the activities of enzymes.

The uptake of sugar by $A$. niger from sucrose solutions was found to be dependent on normal metabolism and hence to be an active process of the same sense of the uptake of hexose. The evidence suggests that the process involved extracellular sucrose breakdown (invertase) to hexose. It also resembles the uptake from mixtures of glucose and fructose in which the glucose moiety was selectively absorbed and an excess of fructose over glucose appeared in the medium. Much more glucose was selectively absorbed; but fructose was supporting agent and made rapid growth

In the following descriptions of maltose utilization which was used to denote the disappearance of maltose from the external solution and degraded sugar in order to release of glucose into environment. Maltose on hydrolysis yields glucose only (maltase). Therefore, the fungus appears to have a higher affinity with glucose. It was observed that spore germination occurred. After spore germination, the cells grew rapidly then the maximum specific growth rates were found on the surface of the plate.

The comparisons among literature and our findings, Yuan et al. (2008), who suggested that A. niger utilize maltose by means of extracellular hydrolysis secretion followed by glucose uptake and metabolism. Salzer and Hager (1991) demonstrated that some fungi could not use sucrose directly but glucose and fructose were readily consumed. Lamb (1974) observed growth of fungi on sucrose, caused by introduction of 'starter' monosaccharide.

Polysaccharides was cleaved extracellularly by means of a broad range of extracellular enzymes (amylases). A. niger was able to grow on all the tested carbon sources e.g.: starch. There were significant differences in the yield of the biomass and amylase production. Degrading of starch was produced mono, di, and oligosaccharides; these were required additional digestion to form available units of absorption. For this reason, growth was becoming slower; meanwhile, the structure of mycelia was less development. These findings are in line with the work conducted by various workers (Omemu et al., 2005; Pandey et al., 2006 and Sasi et al., 2010) where the selection of potent species was made by plate method. However, zonation was correlated with the amount of enzyme produced (amylase) during the isolation of fungi using starch as a sole of carbohydrates.

Generally, among chemical parameters, carbon sources plays a very important role in inducing enzyme secretion and high-affinity of simple sugar transportation and digestion in the medium which was inoculated Aspergillus niger.

\section{CONCLUSION}

In the present study, the growth parameter of $A$. niger was calculated for the observed data showed 
significant variations in the growth rate with different carbon sources (Figure 5). The order of usability of substrate was fructose $>$ glucose $>$ sucrose $>$ maltose $>$ starch. There were recorded high increases in biomass yields and diametric size of colony or mycelia (Table 1).

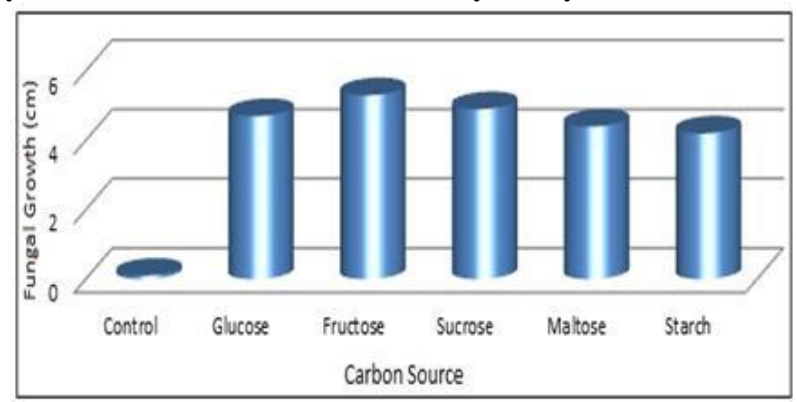

Figure 5. Effect of carbon sources on A. niger, incubation for 5 days at $30{ }^{\circ} \mathrm{C}$ and $\mathrm{pH}$ 5.5.

\section{STATISTICAL ANALYSIS}

The experimental results were carried out in triplicates and it was expressed by mean \pm Standard Error. Statistical analysis was performed using Data Entry: ANOVA; There was considered statistically significant level $p \leq 0.05$.

\section{ACKNOWLEDGMENT}

The authors are grateful to Head of Biology Department at Salahaddin University (Iraq) for providing encouragement and laboratory facilities for carrying out this work.

Table 1. Illustrates the effect of saccharides on the growth of Aspergillus niger

\begin{tabular}{|c|c|c|c|c|c|c|c|c|c|}
\hline \multirow{2}{*}{\multicolumn{2}{|c|}{ Substrates }} & \multicolumn{4}{|c|}{ Sugar Normal Concentration* } & \multicolumn{4}{|c|}{ Sugar Half Concentration* } \\
\hline & & Mean* & SD & Low & $\mathrm{Hi}$ & Mean* & $\mathrm{SD}$ & Low & $\mathrm{Hi}$ \\
\hline \multirow{2}{*}{ Monosaccharide } & Glucose & $4.65 \pm 0.03$ & 0.05 & 4.60 & 4.70 & $3.20 \pm 0.13$ & 0.20 & 3.00 & 3.40 \\
\hline & Fructose & $5.15 \pm 0.10$ & 0.15 & 5.00 & 5.30 & $3.75 \pm 0.13$ & 0.20 & 3.55 & 3.95 \\
\hline \multirow{2}{*}{ Disaccharide } & Maltose & $4.40 \pm 0.13$ & 0.20 & 4.20 & 4.60 & $3.15 \pm 0.20$ & 0.30 & 2.85 & 3.45 \\
\hline & Sucrose & $4.90 \pm 0.06$ & 0.10 & 4.80 & 5.00 & $3.80 \pm 0.06$ & 0.10 & 3.70 & 3.90 \\
\hline Polysaccharide & Starch & $4.00 \pm 0.06$ & 0.10 & 3.90 & 4.10 & $3.30 \pm 0.20$ & 0.30 & 3.00 & 3.60 \\
\hline
\end{tabular}

* Mean = Diameter of colony growth $(\mathrm{cm})$.

* Sugar Normal Concentration = $7.5 \mathrm{~g}$ sugar/ $250 \mathrm{~mL}$ medium; Sugar Half Concentration $=3.75 \mathrm{~g}$ sugar/ $250 \mathrm{~mL}$ medium

* There was significant biologically efficiencies of all substrates $(\mathrm{P} \leq 0.05)$.

\section{REFERENCES}

Adejuwon, A. O., Olanike, O., Olabisi, A., 2012. Production of amylase from Aspergillus niger using defined synthetic growth medium and also rice (Oryza sativa) as growth substrate. E3 Journal of Medical Research, 1 (7): 091-094.

Akroum, S., 2014. Aspergillus niger growth on Polyphenolic carbon source and optimization of the tannase production. G.J.B.B., 3 (3): 246-249.

Bedan, D. S., Aziz, G. M., Al - Sa’ady, A.J.R., 2014. Optimum conditions for $\alpha$ - amylase production by Aspergillus niger mutant isolate using solid state fermentation. Current Research in Microbiology and Biotechnology, 2 (4): 450-456.

Batista, L. R., Silval, D. M., Rezende, E. F., Fungaro, M. P., Alves, E., Sartori, D., 2011. Identification of fungi of the genus Aspergillus section niger using polyphasic taxonomy. Brazilian Journal of Microbiology, 42: 761-773.

Eaton, A. D., Clesceri, L. S., Greenberg, A. E., 1998. Standard Methods for the Examination of Water and Waste water, 20th Ed., American Public Health Association. Washington, USA.

Fang, L., Wenqing, L., Darin, R., Tingyue, G., Murray, M., 1998. Inhibition of extracellular protease secretion by Aspergillus niger using cell immobilization. Biotechnology Letters, 20 (6): 539-542.
Fekete, E., R. P. deVries, Bernhard, S., vanKuyk, P. A., Erzsebet, S., Eva, F., Benjamin, M., Christian, P. K., Levente, K., 2012. D-Galactose uptake is nonfunctional in the conidiophores of Aspergillus niger. Levente Karaffa/ University of Debrecen, 2: 15746968.

Frisvad, J. C., Schuster, E., Dunn-Coleman, N., 2002. On the safety of Aspergillus niger - a review. Apply Microbial Biotechnology, 59: 426-435.

Gupta, V. C., Neha, S. 2012. Batch Biodegradation of Phenol of Paper and Pulp Effluent by Aspergillus niger. International Journal of Chemical Engineering and Applications, 3 (3).

Hashimoto, J. M., Palacios-Cabrera, H., Taniwaki, M. H., Hilary, C. M., 2005. Growth of Aspergillus ochraceus, A. carbonariusand, A. niger on culture media at different water activities and temperatures. Brazilian Journal of Microbiology, 36 (1): 1517-8382.

Henderson, E. K. M., 1961. Isolation, Identification and Growth of some Soil Hyphomycetes and Yeast-Like Fungi which Utilize Aromatic Compounds Related to Lignin. J. Gen. Microbial, 26: 149-154.

Khokhar, I., Mukhtar, I., Mushtaq, S., 2011. Isolation and Screening of Amylolytic Filamentous Fungi. Journal of Applied Science \& Environmental Management, 15 (1): 203-206.

Lamb, R. J., 1974. Effect of D-glucose on utilization of single carbon sources by ectomycorrhizal fungi. Trans. Br. Mycol. Soc., 63 (2): 295-306. 
Maharshi, A. R., Thaker, V. S., 2012. Growth and Development of Plant Pathogenic Fungi in Define Media. European Journal of Experimental Biology, 2 (1): 44-54.

Mehrotra, B. S., Kumar D., 1961. Study on Penicillia. University of Allahabad, 28 (1): 41-48.

Mchunu, N. P., Permau, K., Alam, M., Singh, S., 2013. Carbon utilization profile of a thermophilic fungus, Thermomyces lanuginosus using phenotypic microarray. Advances in Bioscience and Biotechnology, 4: 24-32.

Mcnaught, A. D., 1996. Nomenclature of Carbohydrate. The Royal Society of Chemistry. Thomas Graham House. Science Park. Milton Road. Cambridge. UK.

Omemu, A. M., Akpan, I., Bankole, M. O., Teniola, O. D., 2005. Hydrolysis of raw tuber starches by amylase of Aspergillus niger AM07 isolated from the soil. Afr. J. Biotechnol., 4 (1): 19-25.

Pandey, A., Nigamp, V. T., Socco, L., Singh, D., Mohan, R., 2006. Advances in microbial amylases. Biochem, 31: 35-152

Peña, M. S., 2010. Systems Biology of Glucose Sensing and Repression in Aspergillus niger. $\mathrm{PhD}$ thesis of Chalmers University. Goteborg. Sweden.

Salzer, P., Hager, A., 1991. Sucrose utilization of the ectomycorrhizal fungi Amanita muscaria and Hebeloma crustuliniforme depends on the cell wallbound invertase activity of their host Picea abies. Bot. Acta, 104: 439-445.

Samson, R. A., Hong, S., Frisvad, J. C., 2006. Old and new concepts of species differentiation in Aspergilli. Med. Mycology, 44 (1):133-148.

Sati, S.C., Bisht, S., 2006. Utilization of various carbon sources for the growth of waterborne conidial fungi. The Mycological Society of America, 98 (5): 678 681.

Sasi, A., Kani, M., Panneerselvam, A., Jegadeesh, G., Muthu, K., Kumar, M.R. 2010. Optimizing the conditions of amylase by an Esturian strain of Aspergillus spp. Afr. J. Microbiol. Res., 4 (8): 581586.
Schrickx, J. M., Krave, A. S., Verioes, J. C., Van Den Hondel, C.A.M.J.J, Stouthamer, A. H., Van Verseveld, H. W. 1993. Growth and product formation in chemostat and recycling cultures by Aspergillus niger $\mathrm{N} 402$ and a glucoamylase overproducing transformant, provided with multiple copies of the glaA-gene. Journal of General Microbiology, 139: 2801-2810.

Snellman, C. A., Greathouse, J. E., 1996. The Effect of Glucose on the Growth of Filamentous Fungi in Jet Fuel. Dean of the Faculty. USAF Academy Colorado. USA.

Schuster, E., Dunn-Coleman, N., Frisvad, J., Van Dijck, P., 2002. On the safety of Aspergillus niger - a review. Appl. Microbial Biotechnology, 59: 426-435.

Tandon, R. N., Bilgrami, K. S., 1956. Assimilation of disaccharides by some fungi causing "Leaf Spot" disease, pp. 274-284.

Thyagarajan, R., Raja, S. K., 2010. Influence of carbon source on phytase production by Aspergillus niger. International Journal of Biological Technology, 1 (2): 0976-4313.

Trinci, A.P.J., 1969. A Kinetic Study of the Growth of Aspergillus nidulans and Other Fungi. Journal Gen. Microbial, 57: 11-24.

Walsh, J. H., Harley, J. L., 1962. Sugar absorption by Chaetomium globosum. Department of Agriculture, pp. 299-313.

Yuan, X. L., Van der Kaaij, R. M., Van der Hondel, C., Punt, P. J., Van der Maarel, M., 2008. Aspergillus niger genome-wide analysis reveals a large number of novel alpha-glucan acting enzymes with unexpected expression profiles. Mol. Genet. Genomics, 279: 545561. 